\title{
Modeling the Influence of Technician Proficiency and Maintenance Strategies on Production System Performance
}

\author{
Kai-Wen Tien and Vittaldas Prabhu \\ Pennsylvania State University, University Park PA 16802, United States \\ kut147@psu.edu
}

\begin{abstract}
Maintenance tasks will be the latest automated part of a manufacturing system. Thus, the technician proficiency impacts manufacturing performance. We studied the influence of maintenance proficiency on both machine availability and manufacturing cycle times under three different maintenance strategies: run-to-failure (RTF), preventive maintenance (PM), and condition-based maintenance (CBM). To discuss scenarios in the same framework, we modeled a healthindex based phase-type model and a probability model for imperfect maintenance being subject to technician proficiency. Finally, we simulated a single machine queueing model with different technician proficiency and maintenance strategies. The simulation results revealed that CBM could resist lower proficiency and keep higher availability and low manufacturing cycle times than RTF and PM.
\end{abstract}

Keywords: condition-based maintenance, imperfect maintenance, maintenance proficiency.

\section{Introduction}

Maintenance tasks will be the lastest automated segment of a manufacturing system. The quality of maintenance now still highly depends on the proficiency of maintenance technicians. Moreover, assigning appropriate maintenance technicians is crucial for maintaining productivity and throughput levels. For this purpose, Cabahug et al. (2004) provided a tool to classify maintenance proficiency into three different classes by three factors: years of relevant working experience on the machine, personal disposition, and operator reliability [1]. This classification can help the manager to select the best maintenance operator instead of only resting on manager's intuition and judgment. In addition, Edward et al. (2005) used the artificial neural network (ANN) to classify maintenance proficiency [2]. They claimed that the tool can achieve $89 \%$ accuracy by using ten significant variables.

Condition-based maintenance (CBM) has been studied in many different perspectives. Thanks for the cutting edge technologies such as the Internet of Things (IoTs) and big data analytics, CBM is ready for many different industries. Macchi et al. (2017) came up with a reflection that there is a huge potential for maintenance in this revolution, but maintenance is not yet advanced as it would be expected [3]. To assist 
industries on the pathway of smart manufacturing, Carolis et al. (2017) provided a maturity model for manufacturing companies to assess their digital readiness [4].

In addition, to assess the benefits of $\mathrm{CBM}$, researchers are evaluating the influence of CBM in comparison of preventive maintenance (PM). Yu et al. (2016) provide a maintenance orientated approach to complex system design [5]. They claimed that the approach can reveal many nonobvious interactions between subsystems and produce designs which have lower life-cycle costs compared to traditional approaches. Jonge et al. (2017) reviewed the literature on the relative benefits of CBM over PM on three practical factors of CBM: (1) the effect of required planning time, (2) imperfect condition information, and (3) uncertain failure level. The results showed that the three practical factors could meaningfully impact the relative benefits of CBM to PM [6].

The human element is an unseparated part of maintenance, but only a few studies have discussed the changing of workforce planning during the smart manufacturing era. Koochaki et al. (2013) compare the impact of two strategies - CBM and Preventive Maintenance on workforce planning and scheduling [7]. Their results show that CBM is less efficient than Age-Based Replacement (ABR) on maintenance grouping, but CBM performs better with respect to total maintenance costs.

This work is motivated by the need of capturing the effect of technician proficiency and maintenance strategies on production performance. We investigated the impacts of maintenance proficiency on machine availability and manufacturing cycle times (CT) under three different maintenance strategies: run-to-failure (RTF), preventive maintenance (PM), and condition-based maintenance (CBM). Section 2 provides a phase-type model which describe machine deterioration and failures with machine health index. Moreover, its maintenance cycle time under RTF, PM, and CBM is described. In section 3 , we modeled imperfect maintenance being subjected to technician proficiency. In section 4, we studied maintenance proficiency on machine availability and CTs using a simulation study. The conclusion is made in section 5.

\section{System Description and Assumptions}

\subsection{Health-Index-based Phase-type Deterioration Model}

When studying CBM, we should discuss machine deterioration process. Health index (HI) is a practical tool to represent machine deterioration. It combines the data of operating observations, field inspections, and site and laboratory testing into a quantitative and object index as Fig. 1 (a). Based on observation or other monitored data, the machine states is determined by a range of states from 1 to $N$ (usually 10). HI model is suitable for a complex machine because it merges the senor information into one-dimension values. When IoT is introduced, it is likely to make HI more widespread and systematic rather than ad hoc.

Suppose a machine's HI is continuously monitored, we modeled the HI deterioration process to a phase-type model such as Fig. 1(b). The machine will stay in each HI for a period $1 / \lambda_{i}$ before deteriorating to the next HI. Since, practically, the built HI might not be able to capture all failure modes, we model a Poisson failure rate $\mu_{i}$ in each HI, which represent the uncaptured failure modes, to represent the uncertainty of failure 
level. For example, the second failure in Fig. 1(a) happens before HI achieves the failure state.

When the HI reaches the failure state, the machine will be stopped for maintenance. After maintenance is done, the machine will recover to one of the previous states with probability $\boldsymbol{\alpha}$.Then the time to failure $T_{f}$ can be formulated as a phase-type distribution [8] which distribution function is

$$
F_{T_{f}}(t)=\operatorname{Pr}\left(T_{f} \leq t\right)=1-\boldsymbol{\alpha} \exp (\boldsymbol{S} t) \mathbf{1} .
$$

$\boldsymbol{\alpha}$ is a $N \times 1$ probability array, which represented the probability of the starting state. $\boldsymbol{S}$ is a $N \times N$ sub-generator matrix of the rate matrix. 1 represents an $N \times 1$ vector with every element being 1 .

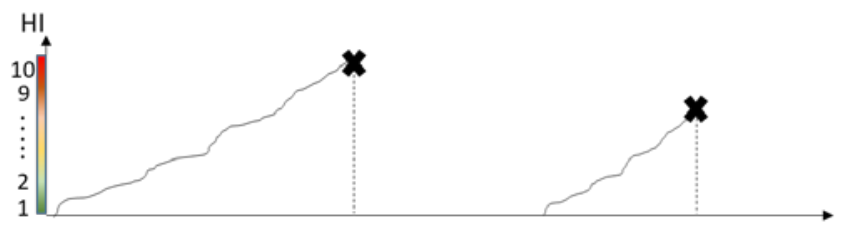

(a)

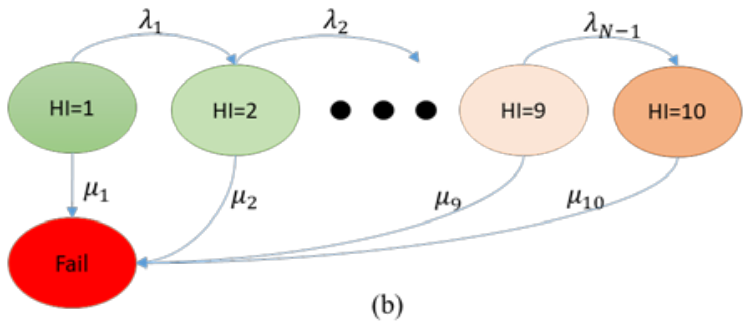

Fig. 1. The sample path of deterioration (a) process is modeled as a phase-type model (b).

\subsection{Maintenance Cycle under Different Strategies}

Once the machine fails, the system will be stopped for "corrective maintenance." After the machine is repaired back to an acceptable condition, the machine will be restarted for production. The function of CBM and PM is to stop the machine in advance to prevent severe failure. However, some minor stoppages might be scheduled to avoid a severe one, which called "planned maintenance."

We define a maintenance cycle is a duration consisting of one time-to-stoppage and its corresponding maintenance time. When PM and CBM come in, the corresponding maintenance cycles are different. Define $R_{i}$ is the time for $i^{t h}$ corrective maintenance; $M_{i}$ is the time for $i^{\text {th }}$ planned maintenance. We assume $\left\{R_{i}, i=1,2 \ldots\right\}$ are i.i.d and $\left\{M_{i}, i=1,2 \ldots\right\}$ are i.i.d.. Usually, the mean and variance of $R_{i}$ is much larger than $M_{i}$. Fig. 1 illustrate a sample path of maintenance cycle. We assume that all sample paths of time-to-failure in the sub-plots are the same. The maintenance cycle is different under different maintenance strategies. 


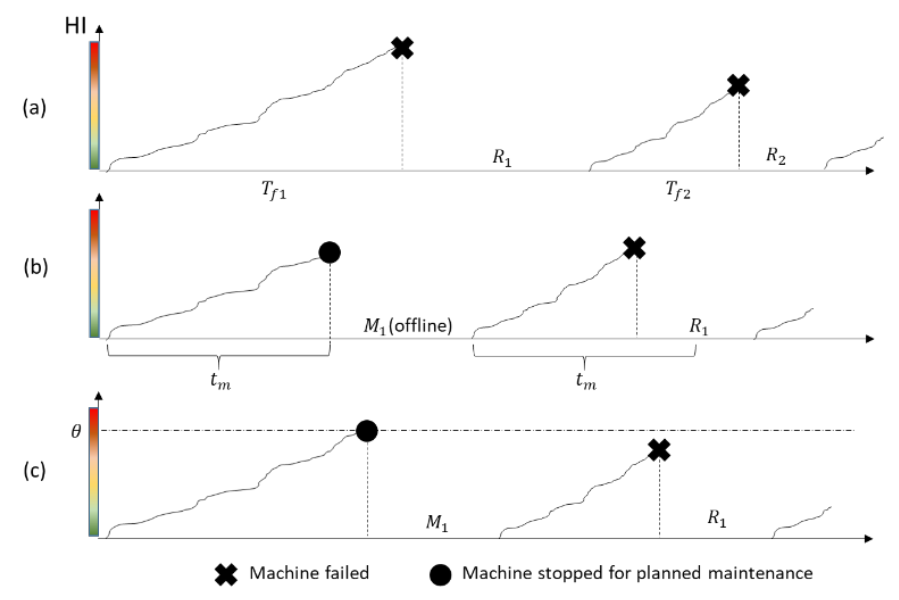

Fig. 2. A sample path of maintenance cycle under (a) RTF, (b) PM, and (c) CBM

RTF strategy as Fig. 2(a) only allow maintenance to operate when the machine breakdown. The uptime in one maintenance cycle is as same as its lifetime distribution. Once the machine breaks down, corrective maintenance will be performed.

PM strategy schedules planned maintenance at a fixed time $t_{m}$ after the last maintenance. In practical applications, PM usually takes place offline (off production time), the planned maintenance time will not affect maintenance cycle times directly. In practice, PM cannot prevent the occurrence of all possible failures since the randomness behavior of failures. Therefore, the uptime in a maintenance cycle is $\min \left\{T_{f}, t_{m}\right\}$. In Fig. 2(b), the first time to failure was longer than $t_{m}$ thus the machine is stopped for planned maintenance. But, the second time to failure was shorter than $t_{m}$ therefore the machine was failed and corrective maintenance came in. In this paper, HI under PM was not used for maintenance decisions.

CBM strategy schedules planned maintenance when HI achieve threshold $\theta$. Ideally, CBM can take place just before the failure. However, practically, CBM cannot prevent the occurrence of all possible failures because of the limitation of technologies or the high cost of monitoring all components. In Fig. 2 (c), the first failure occurred at HI above threshold $\theta$. Therefore, the machine stopped before the failure for planned maintenance. On the other hand, the second failure occurred when HI was lower than $\theta$, so the corrective maintenance came to repair the machine.

\section{$3 \quad$ Modeling imperfect maintenance being subject to Maintenance Proficiency}

Maintenance performance, such as maintenance quality and maintenance time, highly depends on the skill of maintenance technicians. Maintenance proficiency does affect not only the length of maintenance but also maintenance quality. In this study, we as- 
sume that the distribution of maintenance time will not be affected by technician proficiency, but the maintenance is imperfect. Suppose maintenance can recover a machine from 'as new as good' and 'as bad as old.' It implies the HI of the recovered machine is between the old $\mathrm{HI}$ and $\mathrm{HI}=1$. Besides, perfect maintenance will bring a machine back to $\mathrm{HI}=1$.

Suppose the proficiency of maintenance technicians can be evaluated [1, 2, 9]. We defined a maintenance proficiency index $p_{i p f}$, which implies the probability of the machine can be successfully recovered by one $\mathrm{HI}$ from the previous $\mathrm{HI}$ during a maintenance. The probability of the recovered $\mathrm{HI}$ is defined as

$$
P\left(H I_{\text {new }}=i\right)=\left\{\begin{array}{cc}
B\left(i-1 ; H I_{\text {old }}-1,1-p_{\text {ipf }}\right) & i \leq H I_{\text {old }} \\
0 & i>H I_{\text {old }}
\end{array}\right.
$$

$B(x ; n, p)$ is the binomial probability mass function at $x$ given $n$ trials and the probability of success $p . H I_{\text {old }}$ is the HI when maintenance took place; $H I_{\text {new }}$ is the recovered $\mathrm{HI}$ after the machine got maintained. $p_{i p f}$ is defined as probability that $\mathrm{HI}$ ithe $\mathrm{s}$ successfully recovered by one and it is a real value in $(0,1]$. Instances of the distribution is illustrated in Fig. 3. If $p_{i p f}$ is high, the machine is more likely to be repaired back to $\mathrm{HI}=1$. Otherwise, the machine tends to stay in $H I_{\text {old }}$.

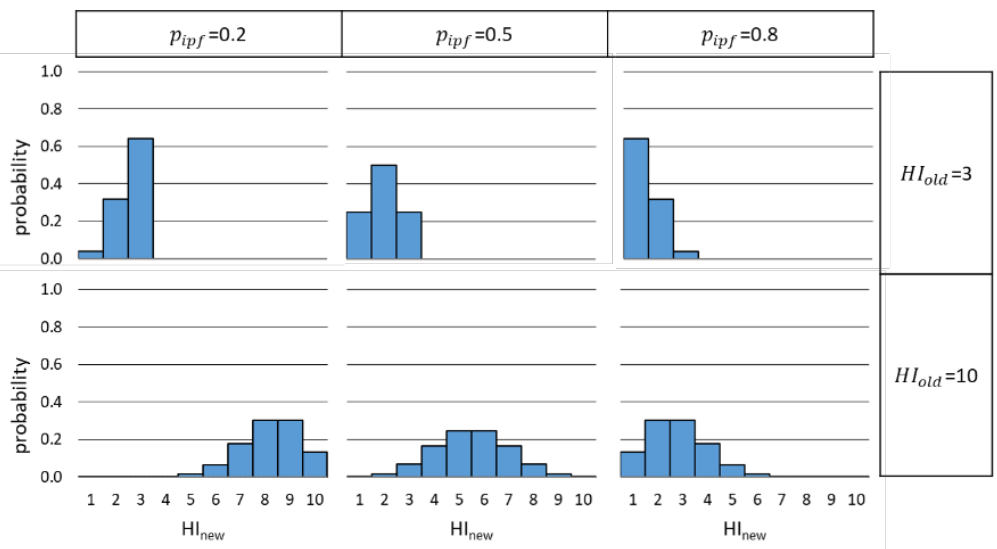

Fig. 3. Distribution of imperfect maintenance being subject to maintenance proficiency

\section{A Simulation Study}

In this section, we discuss the effect of maintenance proficiency on production performance under different maintenance strategies. CT is the time from a job arriving the machine to the job leaving the machine. It usually consists of two parts: queuing time and effective process time. CT is an indicator of production variation. If CT was large, the work-in-progress would be high under the same machine utilization. 


\subsection{Simulation Setting}

We considered a machine which continuously monitored its healthiness. Its health states were scaled from 1 (perfect) to 10 (almost failed). The deterioration and failure rates are shown in Table 1 . Practically, the information can be determined by expert experiences and/or machine history data. Fig. 4 shows that the machine has a tubeshape hazard rate, which is typical for sophisticated machines.

The machine produced one type of product which process time is shown in Table 2. In the simulation, raw material arrival process time is a Poisson process. The machine utilization during available production time is set to be 0.8 , which is common in most of the practical cases. $R_{i}$ and $M_{i}$ were set to be Gamma distributed given mean and variance in Table 2. The simulation had two phases. First, maintenance cycles were simulated by Monte Carlo method using MATLAB ${ }^{\circledR}$. Then the simulated data were imported to the Machine Reliability Logic to a single machine queueing model in Simio ${ }^{\circledR}$. The number of repetitions was set to be 50 , the average of the $95 \%$ half widths over simulation means is less than $10 \%$. Moreover, the simulation is run for 104 weeks (2 years) to generate enough samples since machine failures are rare events. For instance, the number of maintenance cycles under in RTF policy is around 70.

Table 1. Parameters of the Phase-type Model

\begin{tabular}{ccccccccccc}
\hline $\mathrm{HI}$ & 1 & 2 & 3 & 4 & 5 & 6 & 7 & 8 & 9 & 10 \\
\hline$\lambda_{i}(1 / \mathrm{hr})$ & 0.100 & 0.050 & 0.030 & 0.010 & 0.010 & 0.010 & 0.040 & 0.100 & 0.500 & 0.000 \\
\hline$\mu_{i}(1 / \mathrm{hr})$ & 0.010 & 0.005 & 0.002 & 0.001 & 0.001 & 0.001 & 0.003 & 0.800 & 1.200 & 2.000 \\
\hline
\end{tabular}

Table 2. Parameters of Production \& Maintenance Activities

\begin{tabular}{ccccc}
\hline & $\begin{array}{c}\text { Process Time } \\
\text { (hours) }\end{array}$ & $\begin{array}{c}\text { Corrective } \\
\text { Maintenance } \\
\text { Time (hours) }\end{array}$ & $\begin{array}{c}\text { Planned } \\
\text { Maintenance } \\
\text { Time (hours) }\end{array}$ & $\begin{array}{c}\text { Machine Life } \\
\text { Time (hours) }\end{array}$ \\
\hline Mean (hours) & 1.00 & 16.00 & 2.00 & 258.00 \\
\hline Coef. of Variation & 0.00 & 1.00 & 0.50 & 0.75 \\
\hline
\end{tabular}

\subsection{Comparison of Maintenance Strategies}

In this section, we compared the simulation results among different maintenance strategies and proficiency index. Here, $t_{m}$ and $\theta$ were appropriately selected to minimize CT for different $p_{i p f}$. It should be noted that $p_{i p f}=0$, which means the machine cannot recover from current $\mathrm{HI}$ with maintenance, is not realistic. Thus, we do not discuss the value in our simulation study. The results is shown in Fig. 5. RTF was most sensitive to proficiency index. Availability was around $94.10 \%$ and CT was around 6.60 hours. When $p_{\text {ipf }}$ was lower than 0.6, machine availability decreased and CT increased dramatically. On the other hand, the result of PM showed that it can resist imperfect maintenance more than RTF. Availability was around $96.20 \%$ and CT was around 5.5 hours when $p_{i p f} \geq 0.5$. Its performance dropped once $p_{i p f}$ was less than 0.5 . The results of CBM told that CBM could keep high machine availability (96.91\%-96.16\%) and low CT (4.3-1.2 hours) regardless of the proficiency index $p_{\text {ipf }}$. Because HI was 
continuous monitored, the machine stopped when HI excessed the threshold no matter how imperfect maintenance done previously.

It is unsuitable to conclude that CBM always works well in any possible situation because the performance of CBM is highly depended on its health index model. However, it is still worth to understand the effect of maintenance proficiency on production variation under different maintenance strategies with a simulation example. Moreover, we provided insight into modeling maintenance proficiency to compare different maintenance strategies.
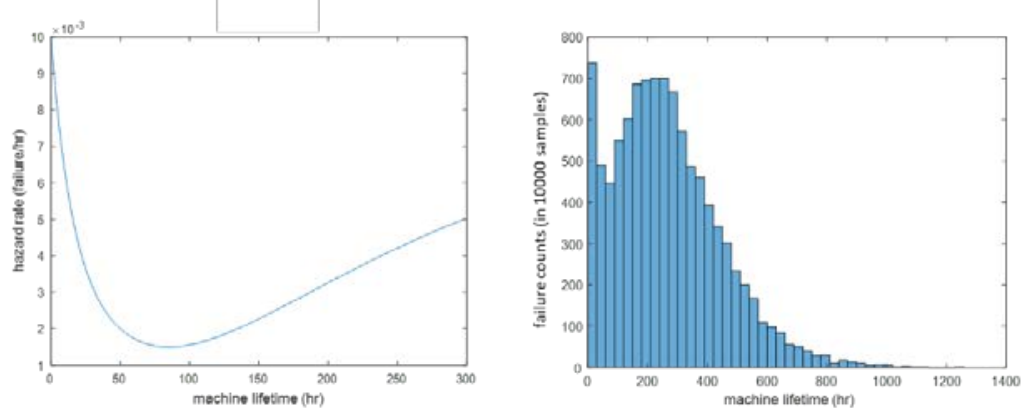

Fig. 4. Hazard function and distribution of time-to-failure


Fig. 5. Comparison of (a) machine availability and (b) manufacturing cycle times according to proficiency indices and maintenance strategies

\section{Conclusion}

This research investigated the impact of maintenance proficiency on production performance under three different maintenance strategies: RTF, PM, and CBM. To equivalently comparing the results, an HI-based phase-type model is proposed. Moreover, to discuss the impact of maintenance proficiency, we proposed a binomial probability model for imperfect maintenance being subject to maintenance proficiency index. Finally, we studied a simulation case. We compare CT and availability under the mainte- 
nance strategies with different proficiency index. The results show CBM was less sensitive than other two strategies. Even when maintenance proficiency is poor ( $p_{i p f}$ is close to zero), the performance of availability and CT were around $96.10 \%$ and 1.20 hours. Since machine under CBM is continuous monitored, a poor maintenance can be detected.

In the future, we will first consider to model learning and forgetting curves into maintenance proficiency index since maintenance technicians will improve their maintenance proficiency when frequent for practicing increases. Secondly, we will study on how to assign maintenance technicians under different strategies or the mix to improve the overall system performance.

\section{References}

1. Cabahug, R.R., Edwards, D.J., Nicholas, J.: Classifying plant operator maintenance proficiency: Examining personal variables, (2004)

2. Edwards, D.J., Yang, J., Cabahug, R., Love, P.E.D.: Intelligence and maintenance proficiency: an examination of plant operators. Constr. Innov. (Sage Publ. Ltd. ). 5, 243-254 (2005)

3. Macchi, M., Roda, I., Fumagalli, L.: On the Advancement of Maintenance Management Towards Smart Maintenance in Manufacturing. In: IFIP Advances in Information and Communication Technology. pp. 383-390. Springer, Cham (2017)

4. De Carolis, A., Macchi, M., Negri, E., Terzi, S.: A maturity model for assessing the digital readiness of manufacturing companies. In: IFIP Advances in Information and Communication Technology. pp. 13-20 (2017)

5. Yu, B.Y., Honda, T., Zubair, S.M., Sharqawy, M.H., Yang, M.C.: A maintenance-focused approach to complex system design. Artif. Intell. Eng. Des. Anal. Manuf. AIEDAM. 30, (2016)

6. de Jonge, B., Teunter, R., Tinga, T.: The influence of practical factors on the benefits of condition-based maintenance over time-based maintenance. Reliab. Eng. Syst. Saf. 158, 21-30 (2017)

7. Koochaki, J., Bokhorst, J.A.C., Wortmann, H., Klingenberg, W.: The influence of condition-based maintenance on workforce planning and maintenance scheduling. Int. J. Prod. Res. 51, 2339-2351 (2013)

8. Buchholz, P., Kriege, J., Felko, I.: Input Modeling with Phase-Type Distributions and Markov Models. SPRINGER BRIEFS IN MATHEMATICS (2014)

9. Edwards, D.J., Holt, G.D., Robinson, B.: An artificial intelligence approach for improving plant operator maintenance proficiency. J. Qual. Maint. Eng. 8, 239252 (2002) 